\title{
Driven by theory
}

\section{Experimentalists working on dissipative self-assembly systems should have a greater appreciation of thermodynamic concepts developed by theoreticians.}

In this issue, we are publishing a Perspective titled 'Dissipative adaptation in driven self-assembly', written by Jeremy England, a thermodynamicist at the Massachusetts Institute of Technology, with the explicit intention of reaching out to experimentalists working on self-assembly.

As chemists are increasingly tackling non-equilibrium supramolecular systems ${ }^{2}$, they are bound to encounter 'emerging' properties at the nanoscale ${ }^{3}$. The explanation of these properties is beyond the limits of many notions of equilibrium thermodynamics that are typically applied in chemistry. Fortunately, theoreticians have been thinking about non-equilibrium and dissipative systems for decades. Ilya Prigogine was, for example, awarded the 1977 Nobel Prize in Chemistry for his contribution to the field. Unfortunately, the complexity of the physics involved, combined with the largely empirical approach of chemistry, has meant that a lot of these concepts have not percolated into the experimental realm. As a journal that strongly supports interdisciplinary research, we are keen to help improve communication between theoreticians and experimentalists, as we have done previously with the field of molecular machines ${ }^{4}$.

The Perspective explains concepts in a manner that experimentalists working on driven self-assembly and dissipative structures should be able to appreciate. Indeed, it was intentionally peer-reviewed by experimentalists, precisely with this goal in mind. The theoretical concepts presented are not new - they have been rigorously reported before in specialized physics literature - but, as England explains, recently there has been a number of theoretical advances that, taken together, might lead towards a more complete understanding of non-equilibrium phenomena. More specifically, the meaning of irreversibility in terms of the amount of work being dissipated as heat as a system moves on a particular trajectory between two states. It turns out, this principle is relatively general, and can be used to explain the self-organization of complex systems such as that observed in nanoscale assemblies, or even in cells.

Moreover, a few recent experimental findings that can be explained in terms of this theoretical framework are discussed. We hope that the Perspective will provide experimentalists with a better appreciation of current thermodynamic conceptualizations and that, in the future, theoretical advances will inspire new experiments in dissipative self-assembly.

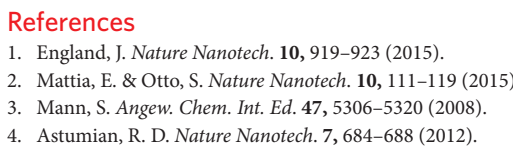

\section{Reporting standards}

\section{A checklist for solar cell articles.}

In January 2015, Nature Nanotechnology introduced a checklist for life sciences manuscripts ${ }^{1}$, which was intended to improve the quality of reporting in these articles. We, together with other Nature journals, are now introducing a reporting checklist for manuscripts on photovoltaic devices (available at http://www.nature.com/ authors/policies/solarchecklist.pdf). We hope that this will, similarly, help improve transparency and reproducibility in the reporting of results on these devices.

The checklist, which has been developed over the last few months through discussions between Nature journal editors and researchers in the community, provides a list of key points regarding the characterization of such devices. These points include the current-voltage scan conditions, the dimensions of the tested solar cells, the number of cells tested, and whether a mask or aperture was used in the measurements; this information should in turn be included in the main text, Methods section or Supplementary Information of the manuscript. We will ask authors of relevant papers to complete the checklist if their work is sent for external peer review, and the completed checklist will be shared with the referees (though it will not be published).

The checklist also asks authors to clarify whether the performance of their device has been independently certified by an institution, such as the National Renewable Energy Laboratory (NREL) or the Fraunhofer Institute for Solar Energy Systems, that tests cells under standard conditions. While certification is not currently a requirement for publication in Nature Nanotechnology, we strongly encourage authors to provide certified efficiency values when possible ${ }^{2}$.

The introduction of this checklist has been driven by concerns in the photovoltaics community over the reporting of device performances and debate over best practices in the field ${ }^{3}$. Concerns have, in particular, been raised over the accuracy of reported power-conversion efficiency values of hybrid organic-inorganic perovskite solar cells ${ }^{4,5}$, issues that have emerged primarily because of a hysteresis in the current-voltage curves of the devices ${ }^{6}$. Care and clarity are key when measuring and reporting the performance of these, and other, photovoltaic devices. The reporting checklist will, we hope, assist in these endeavours.

\footnotetext{
References

1. Nature Nanotech. 9, 949 (2014).

2. Nature Nanotech. 9, 657 (2014)

3. http://www.nature.com/nnano/focus/photovoltaics-performance/ index.html

4. Nature Mater. 13, 837 (2014).

5. Christians, J. A., Manser, J. S. \& Kamat, P. V. J. Phys. Chem. Lett. 6, 852-857 (2015).

6. Snaith, H. et al. J. Phys. Chem. Lett. 5, 1511-1515 (2014).
} 